International Mathematical Forum, Vol. 9, 2014, no. 17, 835 - 847 HIKARI Ltd, www.m-hikari.com http://dx.doi.org/10.12988/imf.2014.4483

\title{
A New Approach to Linear and Nonlinear Schrödinger Equations Using the Natural Decomposition Method
}

\author{
Shehu Maitama \\ Department of Mathematics \\ Northwest University, Kano \\ Kabuga-Katsina Road P.M.B. 3099 Kano State, Nigeria
}

Copyright (c) 2014 Shehu Maitama. This is an open access article distributed under the Creative Commons Attribution License, which permits unrestricted use, distribution, and reproduction in any medium, provided the original work is properly cited.

\begin{abstract}
In this paper, we proposed a new computational algorithms called a new approach to linear and nonlinear Schrödinger equations using the Natural Decomposition Method (NDM). The proposed method is a combination of the Natural transform Method (NTM) and Adomian Decomposition Method (ADM). The new computational algorithms is applied directly without using any transformation, linearization, discretization or taking some restrictive assumptions. Exact solutions of two illustrative examples was successfully fund using the proposed method, and the results are compared with the results of the existing methods. This shows the reliability, efficiency, and accuracy of the proposed method.
\end{abstract}

Mathematics Subject Classification: 35Q55, 35Q40, 35K45

Keywords: Natural Decomposition Method, Adomian polynomials, linear and nonlinear Schrödinger equations

\section{Introduction}

The linear and nonlinear Schrödinger equation are one of the most important partial differential equations in quantum mechanics. Those equations arises in 
the study of the time evolution of the wave function. The linear Schrödinger equation describes the time evolution of a free particle with mass $m$, and its standard form is given by:

$$
v_{t}(x, t)=i v_{x x}, \quad i^{2}=-1, \quad t>0,
$$

subject to the initial condition

$$
v(x, 0)=f(x)
$$

where $f(x)$ is a continuous function and square integrable.

While the nonlinear Schrödinger equation is a solitary wave equation, where the speed of propagation is independent of the amplitude of the wave function, and its standard form is given by:

$$
i v_{t}+v_{x x}+\alpha|v|^{2} v=0
$$

subject to the initial condition

$$
v(x, 0)=f(x)
$$

where $\alpha$ is a constant term and $v(x, t)$ is complex.

Various numerical techniques has been used to solved linear and nonlinear Schrödinger equations, such as Inverse Scattering method [1], Bilinear formalism [2, 3], Adomian Decomposition Method (ADM) [4], Variational Iteration Method (VIM) [4, 5], Laplace Decomposition Method (LDM) [6], Homotopy Perturbation Method (HPM) [7] and so on.

In this article, we proposed a new approach called the Natural Decomposition Method (NDM) for solving linear and nonlinear Schrödinger equations. The new method always lead to exact or approximate solution in form of a rapidly convergence series with elegant computational terms. The proposed method is a combination of the Natural transform method (NTM) and Adomian Decomposition method (ADM). Using the proposed method, we successfully fund exact solutions of two illustrative examples, and the results are compared with the results of the existing methods. This proved the reliability, accuracy and flexibility of the new method. Thus, the Natural Decomposition Method is a powerful mathematical tool for solving linear and nonlinear Schrödinger equations.

The remaining structure of this paper is organized as follows: In Section 2, we begin with the basic definitions and properties of the Natural transform. In section 3, we present the Methodology of the Natural Decomposition Method. In Section 4, we give some numerical applications of the (NDM) to show its effectiveness and accuracy. Finally, section 5 and 6 contained the conclusion and references of this paper. 


\section{Basic Definitions and Properties of the Nat- ural transform}

In this section, we present some definitions and properties of the Natural transform.

Definition: The Natural transform of the function $f(t) \in A$, for $t \in(0, \infty)$ is defined by $[14,15]$ :

$$
\mathbb{N}^{+}[f(t)]=V(s, u)=\int_{0}^{\infty} e^{-s t} f(u t) d t ; s, u \in(0, \infty),
$$

where $\mathbb{N}^{+}[f(t)]$ is the Natural transformation of the time function

$f(t), A=\left\{f(t): \exists M, \tau_{1}, \tau_{2}>0,|f(t)|<M e^{\frac{|t|}{\tau_{j}}}\right.$, if $\left.t \in(-1)^{j} \times[0, \infty)\right\}$ and the variables $s$ and $u$ are the Natural transform variables.

Below, we present some basic theorem of the Natural transform method [14, $15,16]$ :

Theorem 1 If $V(s, u)$ is the Natural transform and $F(s)$ is the Laplace transform of the function $f(t) \in A$, then $\mathbb{N}^{+}[f(t)]=V(s, u)=\frac{1}{u} \int_{0}^{\infty} e^{-\frac{s t}{u}} f(t) d t=$ $\frac{1}{u} F\left(\frac{s}{u}\right)$.

Theorem 2 If $V(s, u)$ is the Natural transform and $G(u)$ is the Sumudu transform of the function $f(t) \in A$, then $\mathbb{N}^{+}[f(t)]=V(s, u)=\frac{1}{s} \int_{0}^{\infty} e^{-t} f\left(\frac{u t}{s}\right) d t=$ $\frac{1}{s} G\left(\frac{u}{s}\right)$.

Theorem 3 If $\mathbb{N}^{+}[f(t)]=V(s, u)$, then $\mathbb{N}^{+}[f(a t)]=\frac{1}{a} V(s, u)$.

Theorem 4 If $\mathbb{N}^{+}[f(t)]=V(s, u)$, then $\mathbb{N}^{+}\left[f^{\prime}(t)\right]=\frac{s}{u} V(s, u)-\frac{f(0)}{u}$.

Theorem 5 If $\mathbb{N}^{+}[f(t)]=V(s, u)$, then $\mathbb{N}^{+}\left[f^{\prime \prime}(t)\right]=\frac{s^{2}}{u^{2}} V(s, u)-\frac{s}{u^{2}} f(0)-$ $\frac{f^{\prime}(0)}{u}$.

Remark The Natural transform is a linear operator. That is, if $\alpha$ and $\beta$ are non-zero constants, then $\mathbb{N}^{+}[\alpha f(t) \pm \beta g(t)]=\alpha \mathbb{N}^{+}[f(t)] \pm \beta \mathbb{N}^{+}[g(t)]=\alpha F^{+}(s, u) \pm \beta G^{+}(s, u)$.

Moreover, $F^{+}(s, u)$ and $G^{+}(s, u)$ are the Natural transforms of $f(t)$ and $g(t)$, respectively. 
Table 1. List of some special Natural transforms and their conversion to Sumudu and Laplace integral transform.

\begin{tabular}{|l|l|l|l|}
\hline$f(t)$ & $\mathbb{N}[f(t)]$ & $\mathbb{S}[f(t)]$ & $\ell[f(t)]$ \\
\hline 1 & $\frac{1}{s}$ & 1 & $\frac{1}{s}$ \\
\hline$t$ & $\frac{u}{s^{2}}$ & $u$ & $\frac{1}{s^{2}}$ \\
\hline$e^{\beta t}$ & $\frac{1}{s-\beta u}$ & $\frac{1}{1-\beta u}$ & $\frac{1}{s-\beta}$ \\
\hline$\frac{t^{n-1}}{(n-1) !}, n=1,2, \ldots$ & $\frac{u^{n-1}}{s^{n}}$ & $u^{n-1}$ & $\frac{1}{s^{n}}$ \\
\hline $\sin (t)$ & $\frac{u}{s^{2}+u^{2}}$ & $\frac{u}{1+u^{2}}$ & $\frac{1}{1+s^{2}}$ \\
\hline
\end{tabular}

\section{Methodology of the Natural Decomposition Method}

In this section, we illustrate the computational algorithm of the Natural Decomposition Method (NDM) to standard nonlinear Schrödinger Eq. (1.3) (1.4):

$$
i v_{t}+v_{x x}+\alpha|v|^{2} v=0
$$

subject to the initial condition

$$
v(x, 0)=f(x)
$$

where $\alpha$ is a constant term and $v(x, t)$ is complex.

We begin by applying the Natural transform on both sides of Eq. (3.1), we obtain:

$$
\frac{s}{u} V(x, s, u)-\frac{1}{u} V(x, 0)-i \mathbb{N}^{+}\left[v_{x x}\right]-i \mathbb{N}^{+}\left[\alpha|v|^{2} v\right]=0 .
$$

Substituting the given initials conditions of Eq. (3.2) into Eq. (3.3), we obtain:

$$
V(x, s, u)=\frac{1}{s} f(x)-\frac{u i}{s} \mathbb{N}^{+}\left[v_{x x}+\alpha|v|^{2} v\right] .
$$

Taking the inverse Natural transform of Eq. (3.4), we obtain

$$
v(x, t)=f(x)+i \mathbb{N}^{-1}\left[\frac{u}{s} \mathbb{N}^{+}\left[v_{x x}+\alpha|v|^{2} v\right]\right],
$$

where $f(x)$ is the source terms, $v(x, t)$ is the unknown function of the nonlinear term $|v(x, t)|^{2} v(x, t)$. 
Now we assume an infinite series solutions for the unknown functions $v(x, t)$ of the form:

$$
v(x, t)=\sum_{n=0}^{\infty} v_{n}(x, t)
$$

Moreover, the nonlinear terms $|v|^{2} v=v^{2} \bar{v}$ can easily be decomposed as follows:

$$
v^{2} \bar{v}=\sum_{n=0}^{\infty} A_{n}
$$

where $A_{n}$ is an Adomian polynomials which can easily be evaluated using the following formula:

$$
A_{n}=\frac{1}{n !} \frac{d^{n}}{d x^{n}}\left[F\left(\sum_{j=0}^{n} \lambda^{j} v_{j}\right)\right]_{\lambda=0}
$$

where $n=0,1,2, \cdots$

Besides, some components of $A_{n}$ are computed below:

$$
\begin{aligned}
A_{0} & =F\left(v_{0}\right), \\
& =v_{0}^{2} \overline{v_{0}}, \\
A_{1} & =v_{1} F^{\prime}\left(v_{0}\right), \\
& =2 v_{0} v_{1} \overline{v_{0}}+v_{0}^{2} \overline{v_{1}}, \\
A_{2} & =v_{2} F^{\prime}\left(v_{0}\right)+\frac{1}{2 !} v_{1}^{2} F^{\prime \prime}\left(v_{0}\right), \\
& =2 v_{0} v_{2} \overline{v_{0}}+v_{1}^{2} \overline{v_{0}}+2 v_{0} v_{1} \overline{v_{1}}+v_{0}^{2} \overline{v_{2}},
\end{aligned}
$$

and so on.

By substituting Eq. (3.6) and Eq. (3.7) into Eq. (3.5), we obtain:

$$
\sum_{n=0}^{\infty} v_{n}(x, t)=f(x)+i \mathbb{N}^{-1}\left[\frac{u}{s} \mathbb{N}^{+}\left[\sum_{n=0}^{\infty} v_{n x x}+\sum_{n=0}^{\infty} A_{n}\right]\right] .
$$

Then by comparing both sides of Eq. (3.9), we can easily generate the recursive relation as follows:

$$
\begin{aligned}
& v_{0}(x, t)=f(x) \\
& v_{1}(x, t)=i \mathbb{N}^{-1}\left[\frac{u}{s} \mathbb{N}^{+}\left[v_{0 x x}+A_{0}\right]\right], \\
& v_{2}(x, t)=i \mathbb{N}^{-1}\left[\frac{u}{s} \mathbb{N}^{+}\left[v_{1 x x}+A_{1}\right]\right], \\
& v_{3}(x, t)=i \mathbb{N}^{-1}\left[\frac{u}{s} \mathbb{N}^{+}\left[v_{2 x x}+A_{2}\right]\right],
\end{aligned}
$$


and so on.

Thus, the general recursive relation of the unknown function $v(x, t)$ is given by:

$$
v_{n+1}(x, t)=i \mathbb{N}^{-1}\left[\frac{u}{s} \mathbb{N}^{+}\left[v_{n x x}+A_{n}\right]\right], n \geq 0 .
$$

Hence, the exact or approximate solutions of the unknown function $v(x, t)$ is given by:

$$
v(x, t)=\sum_{n=0}^{\infty} v_{n}(x, t) .
$$

\section{Applications}

In this section, we apply the Natural Decomposition Method (NDM) to some Linear and Nonlinear Schrödinger equations to show its simplicity, accuracy and reliability.

Example 4.1 Consider the Linear Schrödinger equation of the form:

$$
v_{t}(x, t)=i v_{x x}(x, t)
$$

subject to the initial condition

$$
v(x, 0)=\sin (k x)
$$

where $k$ is constant.

Applying the Natural transform on both sides of Eq. (4.1), we obtain:

$$
\frac{s}{u} V(x, s, u)-\frac{1}{u} V(x, 0)=i \mathbb{N}^{+}\left[v_{x x}(x, t)\right] .
$$

Substituting the given initial condition of Eq. (4.2) into Eq. (4.3), we have:

$$
V(x, s, u)=\frac{\sin (k x)}{s}+i \frac{u}{s}\left[\mathbb{N}^{+}\left[v_{x x}\right]\right] .
$$

Taking the inverse $N$-Transform of Eq. (4.4), we obtain:

$$
v(x, t)=\sin (k x)+i \mathbb{N}^{-1}\left[\frac{u}{s} \mathbb{N}^{+}\left[v_{x x}(x, t)\right]\right] .
$$

We now assume an infinite series solution of the unknown function $v(x, t)$ of the form:

$$
v(x, t)=\sum_{n=0}^{\infty} v_{n}(x, t) .
$$


Then by using Eq. (4.6), we can re-write Eq. (4.5) in the form:

$$
\sum_{n=0}^{\infty} v_{n}(x, t)=\sin (k x)+i \mathbb{N}^{-1}\left[\frac{u}{s} \mathbb{N}^{+}\left[\sum_{n=0}^{\infty} v_{n x x}(x, t)\right]\right] .
$$

Comparing both sides of Eq. (4.7), we can easily generate the recursive relation as follows:

$$
\begin{aligned}
& v_{0}(x, t)=\sin (k x) \\
& v_{1}(x, t)=i \mathbb{N}^{-1}\left[\frac{u}{s} \mathbb{N}^{+}\left[v_{0 x x}(x, t)\right]\right], \\
& v_{2}(x, t)=i \mathbb{N}^{-1}\left[\frac{u}{s} \mathbb{N}^{+}\left[v_{1 x x}(x,, t)\right]\right], \\
& v_{3}(x, t)=i \mathbb{N}^{-1}\left[\frac{u}{s} \mathbb{N}^{+}\left[v_{2 x x}(x, t)\right]\right],
\end{aligned}
$$

and so on.

Thus, the general recursive relation is given by:

$$
v_{n+1}(x, t)=i \mathbb{N}^{-1}\left[\frac{u}{s} \mathbb{N}^{+}\left[v_{n x x}(x, t)\right]\right], \quad \forall n \geq 0
$$

Using the general recursive relation, we can easily compute the remaining components of the unknown function $(x, t)$ as follows:

$$
\begin{aligned}
v_{1}(x, t) & =i \mathbb{N}^{-1}\left[\frac{u}{s} \mathbb{N}^{+}\left[v_{0 x x}(x, t)\right]\right] \\
& =i \mathbb{N}^{-1}\left[\frac{u}{s} \mathbb{N}^{+}[\sin (k x)]\right] \\
& =i \sin (k x) \mathbb{N}^{-1}\left[\frac{u}{s} \mathbb{N}^{+}[1]\right] \\
& =\sin (k x) \mathbb{N}^{-1}\left[\frac{u}{s^{2}}\right] \\
& =(i t) \sin (k x), \\
v_{2}(x, t) & =i \mathbb{N}^{-1}\left[\frac{u}{s} \mathbb{N}^{+}\left[v_{1 x x}(x, t)\right]\right] \\
& =i \mathbb{N}^{-1}\left[\frac{u}{s} \mathbb{N}^{+}[i t \sin (k x)]\right] \\
& =-\sin (k x) \mathbb{N}^{-1}\left[\frac{u}{s} \mathbb{N}^{+}[t]\right] \\
& =-\sin (k x) \mathbb{N}^{-1}\left[\frac{u^{2}}{s^{3}}\right] \\
& =\frac{(i t)^{2}}{2 !} \sin (k x),
\end{aligned}
$$




$$
\begin{aligned}
v_{3}(x, t) & =i \mathbb{N}^{-1}\left[\frac{u}{s} \mathbb{N}^{+}\left[v_{2 x x}(x, t)\right]\right] \\
& =i \mathbb{N}^{-1}\left[\frac{u}{s} \mathbb{N}^{+}\left[-\frac{t^{2}}{2 !} \sin (k x)\right]\right] \\
& =-\frac{i \sin (k x)}{2 !} \mathbb{N}^{-1}\left[\frac{u}{s} \mathbb{N}^{+}\left[t^{2}\right]\right] \\
& =-\frac{i}{2 !} \sin (k x) \mathbb{N}^{-1}\left[\frac{2 u^{3}}{s^{4}}\right] \\
& =\frac{(i t)^{3}}{3 !} \sin (k x)
\end{aligned}
$$

and so on.

Hence, the series solution of the unknown function $v(x, t)$ is given by:

$$
\begin{aligned}
v(x, t) & =\sum_{n=0}^{\infty} v_{n}(x, t) \\
& =v_{0}(x, t)+v_{1}(x, t)+v_{2}(x, t)+v_{3}(x, t)+\cdots \\
& =\sin (k x)+(i t) \sin (k x)+\frac{\left(i t^{2}\right)^{2}}{2 !} \sin (k x)+\frac{(i t)^{3}}{3 !} \sin (k x)+\cdots \\
& =\sin (k x)\left(1+(i t)+\frac{(i t)^{2}}{2 !}+\frac{(i t)^{3}}{3 !}+\cdots\right) .
\end{aligned}
$$

Thus, the exact solution of the Schrodinger equation (4.1)-(4.2) is given by:

$$
v(x, t)=\sin (k x) e^{i t} .
$$

The exact solution is in closed agreement with the result obtained by (ADM) [4] and (VIM) [4, 5].

Example 4.2 Consider the following nonlinear Schrödinger equation of the form:

$$
i v_{t}+v_{x x}+6|v|^{2} v=0
$$

subject to the initial condition

$$
v(x, 0)=e^{3 i x} .
$$

Taking the Natural transform on both sides of Eq. (4.9), we obtain:

$$
\frac{s}{u} V(x, s, u)-\frac{1}{u} V(x, 0)-i \mathbb{N}^{+}\left[v_{x x}\right]-6 i \mathbb{N}^{+}\left[|v|^{2} v\right]=0
$$

Then by substituting the given initial condition of Eq. (4.10), into Eq. (4.11), we obtain:

$$
V(x, s, u)=\frac{e^{3 i x}}{s}+i \frac{u}{s} \mathbb{N}^{+}\left[v_{x x}+6|v|^{2} v\right]=0
$$


By taking the inverse Natural transform of Eq. (4.12), we obtain:

$$
v(x, t)=e^{3 i x}+i \mathbb{N}^{-1}\left[\frac{u}{s} \mathbb{N}^{+}\left[v_{x x}+6|v|^{2} v\right]\right] .
$$

We now assume an infinite series solution of the unknown function $v(x, t)$ of the form:

$$
v(x, t)=\sum_{n=0}^{\infty} v_{n}(x, t)
$$

Using Eq. (4.14), we can re-write Eq. (4.13) in the form:

$$
\sum_{n=0}^{\infty} v_{n}(x, t)=e^{3 i x}+i \mathbb{N}^{-1}\left[\frac{u}{s} \mathbb{N}^{+}\left[\sum_{n=0}^{\infty} v_{n x x}+6 \sum_{n=0}^{\infty} A_{n}\right]\right]
$$

where $A_{n}$ is an Adomian polynomial which represent the nonlinear term $|v|^{2} v$ By comparing both sides of Eq. (4.15), we can easily generate the recursive relation as follows:

$$
\begin{aligned}
v_{0}(x, t) & =e^{3 i x}, \\
v_{1}(x, t) & =i \mathbb{N}^{-1}\left[\frac{u}{s} \mathbb{N}^{+}\left[v_{0 x x}+6 A_{0}\right]\right], \\
v_{2}(x, t) & =i \mathbb{N}^{-1}\left[\frac{u}{s} \mathbb{N}^{+}\left[v_{1 x x}+6 A_{1}\right]\right], \\
v_{3}(x, t)= & =i \mathbb{N}^{-1}\left[\frac{u}{s} \mathbb{N}^{+}\left[v_{2 x x}+6 A_{2}\right]\right] .
\end{aligned}
$$

Continuing in this same manner, we will eventually obtain:

$$
v_{n+1}(x, t)=i \mathbb{N}^{-1}\left[\frac{u}{s} \mathbb{N}^{+}\left[v_{n x x}+6 A_{n}\right]\right], \quad \forall n \geq 0 .
$$

Therefore, using the general recursive relation of Eq. (4.16), we can easily 
compute the remaining components of the unknown function $v(x, t)$ as follows:

$$
\begin{aligned}
& v_{1}(x, t)=i \mathbb{N}^{-1}\left[\frac{u}{s} \mathbb{N}^{+}\left[v_{0 x x}+A_{0}\right]\right] \\
& =i \mathbb{N}^{-1}\left[\frac{u}{s} \mathbb{N}^{+}\left[v_{0 x x}+6 v_{0}^{2} \bar{v}\right]\right] \\
& =i \mathbb{N}^{-1}\left[\frac{u}{s} \mathbb{N}^{+}\left[-3 e^{3 i x}\right]\right] \\
& =-3 i e^{3 i x} \mathbb{N}^{-1}\left[\frac{u}{s} \mathbb{N}^{+}[1]\right] \\
& =-3 i e^{3 i x} \mathbb{N}^{-1}\left[\frac{u}{s^{2}}\right] \\
& =-(3 i) t e^{3 i x} \text {, } \\
& v_{2}(x, t)=i \mathbb{N}^{-1}\left[\frac{u}{s} \mathbb{N}^{+}\left[v_{1 x x}+6 A_{1}\right]\right] \\
& =i \mathbb{N}^{-1}\left[\frac{u}{s} \mathbb{N}^{+}\left[v_{1 x x}+6\left(2 v_{0} v_{1} \overline{v_{0}}+v_{0}^{2} v_{1}\right)\right]\right] \\
& =i \mathbb{N}^{-1}\left[\frac{u}{s} \mathbb{N}^{+}\left[9 i t e^{3 i x}\right]\right] \\
& =-9 e^{3 i x} \mathbb{N}^{-1}\left[\frac{u}{s} \mathbb{N}^{+}[t]\right] \\
& =-9 e^{3 i x} \mathbb{N}^{-1}\left[\frac{u^{2}}{s^{3}}\right] \\
& =\frac{(3 i t)^{2}}{2 !} e^{3 i x} \text {, } \\
& v_{3}(x, t)=i \mathbb{N}^{-1}\left[\frac{u}{s} \mathbb{N}^{+}\left[v_{2 x x}+6 A_{2}\right]\right] \\
& =i \mathbb{N}^{-1}\left[\frac{u}{s} \mathbb{N}^{+}\left[v_{1 x x}+6\left(2 v_{0} v_{2} \overline{v_{0}}+v_{1}^{2} \overline{v_{1}}+2 v_{0} v_{1} \overline{v_{1}}+v_{0}^{2} \overline{v_{2}}\right)\right]\right] \\
& =i \mathbb{N}^{-1}\left[\frac{u}{s} \mathbb{N}^{+}\left[\frac{27 t^{2}}{2 !} e^{3 i x}\right]\right] \\
& =\frac{27 i}{2 !} e^{3 i x} \mathbb{N}^{-1}\left[\frac{u}{s} \mathbb{N}^{+}\left[t^{2}\right]\right] \\
& =\frac{27 i}{2 !} e^{3 i x} \mathbb{N}^{-1}\left[\frac{2 u^{3}}{s^{4}}\right] \\
& =-\frac{(3 i t)^{3}}{3 !} e^{3 i x}
\end{aligned}
$$

and so on. 
Hence, the series solution of the unknown function $v(x, t)$ is given by:

$$
\begin{aligned}
v(x, t) & =\sum_{n=0}^{\infty} v_{n}(x, t) \\
& =v_{0}(x, t)+v_{1}(x, t)+v_{2}(x, t)+v_{3}+\cdots \\
& =e^{3 i x}-3 i t e^{3 i x}+\frac{(3 i t)^{2}}{2 !} e^{3 i x}+\cdots \\
& =e^{3 i x}\left(1-3 i t+\frac{(3 i t)^{2}}{2 !}-\frac{(3 i t)^{3}}{3 !} \cdots\right) \\
& =e^{3 i(x-t)}
\end{aligned}
$$

Therefore, the exact solution of the Schrodinger equation (4.9)-(4.10) is given by:

$$
v(x, t)=e^{3 i(x-t)} .
$$

The exact solution is in closed agreement with result obtained by (ADM) [4] and (VIM) [4, 5].

\section{Conclusion}

The aim of this article is to introduce a new computational algorithm called the Natural Decomposition Method for solving linear and nonlinear Schrödinger equation without any linearization, discritization, transformation, or taking some restrictive assumptions. Exact solutions of two illustrative examples was successfully fund and the results are compared with (ADM) [4] and (VIM) [4, 5]. This shows the simplicity, efficiency and accuracy of the new approach. Thus, the Natural Decomposition Method (NDM) can easily be use to solve many linear and nonlinear Schrödinger equations and related applications in applied mathematics and engineering.

\section{Acknowledgement}

The author would like to thank the editor, anonymous referees' for their comments and suggestions in this article, and the Executive Governor of Kano State, (Dr.) Rabiu Musa Kwankwaso for funding the research.

\section{References}

[1] M. Ablowitz and H. Segur, Solitons and the Inverse Scattering Transform, SIAM , (1981). 
[2] R. Hirota, Direct method in soliton theory, In Solitons (Bullogh, R.K. and Caudrey, P, J., eds), Springer, Berlin (1980).

[3] R. Hirota, Exact N-soliton solutions of the wave equation of long waves in shallow-water and in nonlinear lattices, Journal of Maths. Phys., 14 (7) (1973), 810-814.

[4] A.M. Wazwaz, Partial Differential Equations and Solitary Waves Theory, Springer-Verlag, Heidelberg (2009).

[5] N. H. Sweilam, Variational Iteration Method for Solving Cubic nonlinear Schrodinger Equation, Journal of Computational and Applied Mathematics, 207 (2007), 155-163.

[6] Arun Kumar and R. D. Pankaj, Solitary Wave Solutions of Schrödinger Equation by Laplace-Adomian Decomposition Method, Physical Review and Reseach International, 3(4) (2013), 702-712.

[7] J. Biazar, H. Ghazvini, Exact solution for nonlinear Schrödinger equation by Hes Homotopy Pertubation Method, Phys. Lett. A, in press, doi:10.1016j.physlet., (2007).

[8] Arun Kumar, A Variational Description of Nonlinear Schrödinger Equation, International Journal of Computational and Applied Mathematics., 450 (2002), 201-205.

[9] . Adomian, Solving frontier problems of physics: the decomposition method, Kluwer Acad. Publ (1994).

[10] G. Adomian, A new approach to nonlinear partial differential equations, J. Math. Anal. Appl., 102 (1984), 420-434.

[11] El-sayed, The decomposition method for studying the Klein-Gordon Equation, Chaos, Soliton and Fractals , 18 (2003), 1025-1030.

[12] J. Biarzar, E. Babolian, R. Islam, An alternative algorithm for computing Adomian polynomial in special cases, Applied Mathematics and Computation, 138 (2003), 523-529.

[13] Wazwaz A., The modified decomposition method for analytic treatment of differential equations, Applied Mathematics and Computations, 173 (2006), 165-176.

[14] Fethi Bin. Muhammed Belgacem, R. Silambarasan, Theory of Natural Transform, Mathematical Engineering, Science and Aerospace., 3 (1)(2012), 100-124. 
[15] F.B.M. Belgacem, R. Silambarasan, Maxwell's equations by means of the natural transform, Mathematics in Engineering, Science and aerospace, 3 (2012), 313-323.

[16] Z.H. Khan, W.A. Khan, N-transform properties and applications, NUST Jour. of Engg. Sciences, 1 (2008), 127-133.

[17] Hussain, M. Khan, A variationa iteration method for solving linear and nonlinear Klein-Gordon equation, Applied Mathematical Science, 4 (2010), 1931-1940.

[18] F.B.M. Belgacem, R. Silambarasan, Theoretical investigations of the natural transform, Progress In Electromagnetics Research Symposium Proceedings, Suzhou, China, Sept. 12 (16) (2011).

[19] F.B.M. Belgacem, R. Silambarasan, Maxwell's equations solutions through the natural transform, Mathematics in Engineering, Science and Aerospace 3 (3) (2012), 313-323.

[20] M.G.M. Hussain, F.B.M. Belgacem, Transient solutions of Maxwell's equations based on Sumudu transform, Progress in Electromagnetics Research, 74 (2007), 273-289.

[21] M.R. Spiegel, Theory and Problems of Laplace Transforms, Schaums Outline Series, McGraw-Hill, New York (1965).

[22] S.T. Khuri, A Laplace decomposition algorithm applied to a class of nonlinear partial differential equations, Journal of Applied Mathematics, 1 (2001), 141-155.

[23] Belgacem, F. B. M., Karaballi, A. A., Kalla, S. L.; Analytical investigations of the Sumudu transform and applications to integral production equations, Mathematical Problems in Engineering, 3 (2003), 103-118.

[24] Belgacem, F. B. M., Karaballi, A. A., Sumudu transform fundamental properties, investigations and applications, Journal of Applied Mathematics and Stochastic Analysis, 40 (2006), 1-23.

\section{Received: April 25, 2014}

